Original paper

\title{
Radiomics analysis of EPID measurements for patient positioning error detection in thyroid associated ophthalmopathy radiotherapy
}

\author{
Xiangbin Zhang ${ }^{1}$, Guyu Dai ${ }^{1}$, Renming Zhong, Li Zhou, Qing Xiao, Xuetao Wang, Jialu Lai, \\ Jianling Zhao, Guangjun $\mathrm{Li}^{\prime \prime}$, Sen Bai \\ Department of Radiation Oncology, Cancer Center and State Key Laboratory of Biotherapy, West China Hospital, Sichuan University, Chengdu, Sichuan 610041, China
}

\section{A R T I C L E I N F O}

\section{Keywords:}

EPID dosimetry

Positioning error detection

Multifactorial error sources

Radiomics analysis

Machine learning

\begin{abstract}
A B S T R A C T
Purpose: Electronic portal imaging detector (EPID)-based patient positioning verification is an important component of safe radiotherapy treatment delivery. In computer simulation studies, learning-based approaches have proven to be superior to conventional gamma analysis in the detection of positioning errors. To approximate a clinical scenario, the detectability of positioning errors via EPID measurements was assessed using radiomics analysis for patients with thyroid-associated ophthalmopathy.

Methods: Treatment plans of 40 patients with thyroid-associated ophthalmopathy were delivered to a solid anthropomorphic head phantom. To simulate positioning errors, combinations of 0-, 2-, and 4-mm translation errors in the left-right (LR), superior-inferior (SI), and anterior-posterior (AP) directions were introduced to the phantom. The positioning errors-induced dose differences between measured portal dose images were used to predict the magnitude and direction of positioning errors. The detectability of positioning errors was assessed via radiomics analysis of the dose differences. Three classification models-support vector machine (SVM), k-nearest neighbors (KNN), and XGBoost-were used for the detection of positioning errors (positioning errors larger or smaller than $3 \mathrm{~mm}$ in an arbitrary direction) and direction classification (positioning errors larger or smaller than $3 \mathrm{~mm}$ in a specific direction). The receiver operating characteristic curve and the area under the ROC curve (AUC) were used to evaluate the performance of classification models.

Results: For the detection of positioning errors, the AUC values of SVM, KNN, and XGBoost models were all above 0.90. For LR, SI, and AP direction classification, the highest AUC values were 0.76, 0.91, and 0.80, respectively. Conclusions: Combined radiomics and machine learning approaches are capable of detecting the magnitude and direction of positioning errors from EPID measurements. This study is a further step toward machine learningbased positioning error detection during treatment delivery with EPID measurements.
\end{abstract}

\section{Introduction}

Modern radiotherapy, with highly conformal dose distributions, is quite susceptible to positioning errors. Thus, to ensure safety, patient positioning consistency check is required during actual delivery $[1,2]$. A well-established patient positioning in vivo verification approach during radiotherapy delivery is electronic portal imaging device (EPID) dosimetry.

Several studies have explored the sensitivity of EPID dosimetry in patient positioning verification [3-5]. These usually employ gamma indices extracted from conventional gamma analysis of EPID dosimetry as evaluation metrics. However, the extraction of gamma indices neglects the detailed information of gamma distribution [6,7]. This compresses the information of EPID dosimetry, decreases the potential of error source identification.

Learning-based approaches have recently proven to be superior to conventional gamma analysis in EPID dosimetry analysis [8-11]. Nyflota et al. [8] and Wootton et al. [9] explored the use of learningbased approaches for multi-leaf collimator (MLC) mispositioning error detection. Ma et al. [10] further demonstrated the potential of radiomics analysis in MLC mispositioning error detection. Wolfs et al. [11] in their simulation study validated the powerful of deep learning for various treatment errors identification. These studies confirmed that learningbased approaches are useful in providing additional information not

\footnotetext{
* Corresponding authors.

E-mail addresses: gjnick829@sina.com (G. Li), baisen@scu.edu.cn (S. Bai).

1 These authors contributed equally to this work.
} 
currently available from EPID dosimetry.

Collectively, most of these studies focused on pretreatment verification (MLC mispositioning error detection), in which a single factorial error source simulation is sufficient. However, in on-treatment scenarios, multifactorial error sources are common. To illustrate this, positioning errors are always mixed with linac mechanical errors. For the purpose of checking patient positioning consistency, simulating a single factorial error source is of limited utility, and multifactorial error sources simulation is necessary. Radiomics analysis is generally used for transferring medical imaging to higherdimensional data and mining of these data for improved decision support $[12,13]$. Combined with machine learning approaches, radiomics analysis has the potential to extract useful information from EPID dosimetry for multifactorial error sources identification.

The aim of this study was to analyze the detectability of positioning errors through radiomics analysis of EPID measurements from patients with thyroid-associated ophthalmopathy. Thyroid-associated ophthalmopathy is a benign disease that occurs adjacent to the radiosensitive eye lenses and can be addressed through radiotherapy [14,15]. Therefore, this treatment requires accurate positioning for eye lenses sparing, and low-frequency cone-beam CT-based image guidance for imaging dose reduction. For these reasons, EPID-based in vivo verification of patient positioning is particularly important in thyroidassociated ophthalmopathy radiotherapy.

To approximate a clinical scenario and enable multifactorial error source simulation, in this study, positioning errors were introduced during actual treatment delivery. The detectability of these positioning errors through the radiomics analysis of EPID measurements was then analyzed from two perspectives: clinically relevant positioning error in an arbitrary direction (the detection of positioning errors), and clinically relevant positioning error in a specific direction (direction classification). More specifically, the detection of positioning errors was used to identify the magnitudes of the positioning errors, and direction classification was used to classify the magnitudes and directions of the errors.

\section{Materials and Methods}

\subsection{Patients information and error simulation}

Data from 40 patients with thyroid-associated ophthalmopathy were enrolled in this study. All patients underwent volumetric modulated arc therapy (VMAT) with a prescription dose of 20 Gy delivered over 10 fractions. The dual arc VMAT plans (clockwise $240^{\circ}-120^{\circ}$ and anticlockwise $120^{\circ}-240^{\circ}$ ) were optimized using photon optimizer algorithm and calculated using anisotropic analytical algorithm in Eclipse system (v13.6, Varian Medical Systems, Palo Alto, CA). According to our clinical setting, the slice thickness of planning CT (SOMATOM Definition AS+, Siemens Medical Solutions, Forchheim, Germany) was $3 \mathrm{~mm}$. The origins to insertions of the extra-ocular muscles and the retroorbital fatty spaces with the main bulk were defined as the clinical target volume (CTV). The lenses, globes, optic nerves, and lacrimal glands were defined as organs at risk (OARs). The planned target volume (PTV) is the clinical target volume (CTV) plus $3 \mathrm{~mm}$ in all directions. Before the first treatment fraction, cone-beam CT image guidance was performed to verify the planned patient position.

To approximate a clinical scenario, these clinical planned doses were delivered to a solid anthropomorphic head phantom (Chengdu Dosimetric Phantoms, Chengdu, China), and portal dose image (PDI) was acquired on a Varian Edge radiosurgery system (Varian Medical Systems, Palo Alto, CA). The solid anthropomorphic head phantom (Fig. 1) represented a model of an adult human male skull embedded in a soft tissue-equivalent synthetic material to match the attenuation and scattering properties of the bone, soft tissue, and airways in the human head [16].

To simulate positioning errors, the solid anthropomorphic head phantom was translated using 27 combinations of $0-, 2-$, and 4-mm translation positioning errors in left-right (LR), superior-inferior (SI), and anterior-posterior (AP) axis. Notably, the translation positioning errors were simulated in a single direction along every axis. To eliminate the uncertainty induced by $2 \mathrm{D}$ dose prediction model [17], the measured PDIs without and with positioning error were defined as PDI $\mathrm{I}_{\text {ref }}$ and $\mathrm{PDI}_{\text {comp }}$, respectively, and $\mathrm{PDI}_{\text {diff }}$ was then acquired by subtracting

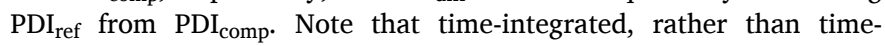
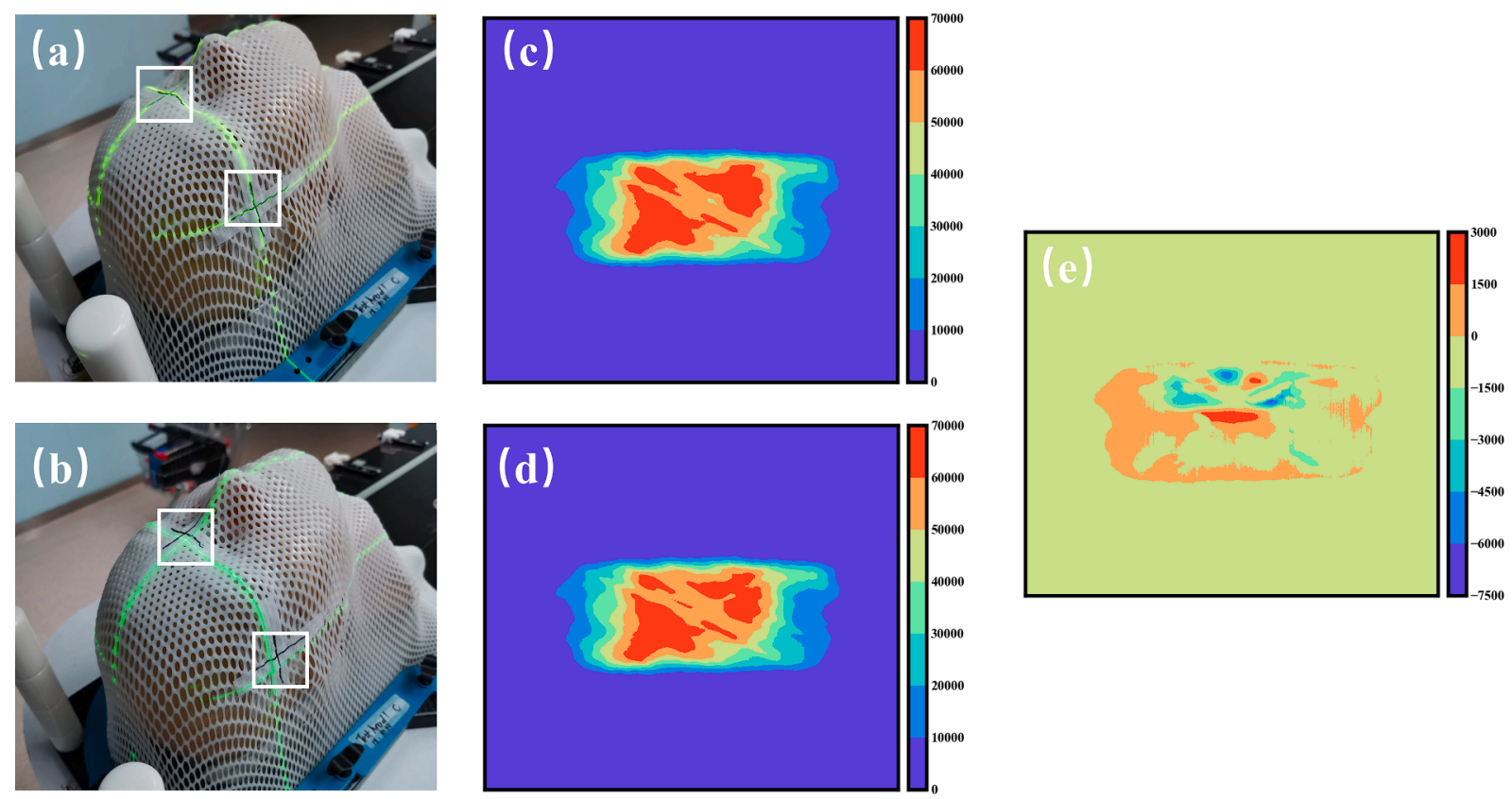

Fig. 1. Example of PDI acquisition with a solid anthropomorphic head phantom. a) The solid anthropomorphic head phantom without positioning errors. b) The solid anthropomorphic head phantom with positioning errors. c) The acquired PDI ref $_{\text {without positioning errors. d) The acquired PDI }}$ comp with positioning errors. e) The resulting $\mathrm{PDI}_{\text {diff }}$ induced by positioning errors. 
resolved, PDIs were analyzed in this study.

\subsection{Classification of clinically relevant positioning errors}

For clinically relevant positioning errors classification, each $\mathrm{PDI}_{\text {diff }}$ was assigned four labels with two classification aspects, as shown in Fig. 2. Positioning errors exceeding $3 \mathrm{~mm}$, which is equal to the PTV margin used in this study, were defined as clinically relevant. The detection of positioning errors involves the assessment of clinically relevant positioning errors in an arbitrary direction, whereas direction classification involves the assessment of clinically relevant positioning errors in a specific direction-in this context, the LR, SI, and AP directions (three classification tasks).

\subsection{Radiomics features extraction and selection}

Fig. 3 shows the radiomics analysis workflow for positioning errors detection with EPID measurements. A total of 94 radiomics features were extracted from PDI $_{\text {diff }}$ using the pyradiomics package [13]. The extracted radiomics features included first-order (19 features), graylevel co-occurrence matrix (GLCM, 24 features), gray-level size zone matrix (GLSZM, 16 features), gray-level run length matrix (GLRLM, 16 features), neighboring gray tone difference matrix (NGTDM, five features), and gray-level dependence matrix (GLDM, 14 features) groups. Because the shapes of the PDIs remained constant between treatment fractions, the radiomics features of shape (2D) and shape (3D) groups were excluded from this analysis.

For each classification task, Pearson correlational analysis of each radiomics feature and classification label was done to eliminate the uncorrelated features $(r<0.1, P>0.05)$, using the scipy package [18]. The results of this correlational analysis are provided in Supplementary Material A. Principal component analysis was then performed to avoid multicollinearity in the selected radiomics features. These transformed features were then used as inputs for the radiomics analysis. Notably, feature selection, model optimization, and model evaluation were implemented using the scikit-learn package [18].

\subsection{Model optimization and evaluation}

Data from 32 patients (864 $\mathrm{PDI}_{\text {diff }}$ ) were used for model optimization, and data from 8 patients $\left(216 \mathrm{PDI}_{\text {diff }}\right.$ ) were used for model evaluation. To enable cross-validation, the 32 patients ( $864 \mathrm{PDI}_{\text {diff }}$ ) were split into training and validation data sets using the leave-one-out method. Support vector machine (SVM), k-nearest neighbors (KNN), and XGBoost [19] classification models were then utilized to find the optimal classifier for each classification task. The hyperparameters for the model optimization are listed in Supplementary Material B.

The performance of the classification models on the training, validation, and test datasets were assessed using the F1-score, or harmonic mean of the precision and recall, which is suitable for evaluating the results of the class imbalance learning in our study. The F1-score ranges from 0 to 1 , which represents worst to perfect, respectively. For the test datasets, the receiver operating characteristic curve (ROC curve) and the area under the ROC curve (AUC) were obtained to assess the performance of the classification models in detail.

Precision $=\mathrm{TP} /(\mathrm{TP}+\mathrm{FP})$

Recall $=\mathrm{TP} /(\mathrm{TP}+\mathrm{FN})$

F1 - score $=2 \times$ Precision $\times$ Recall $/($ Precision + Recall $)$

TP, FP, and FN refer to true positives, false positives, and false negatives, respectively.

\section{Results}

The F1-scores of the training, validation, and test datasets are listed in Table 1. The differences between the results of the validation and test datasets range from 0.004 to 0.031 , indicating the robustness of the fitted models. Additionally, the performance of the SVM, KNN, and XGBoost models are comparable.

For the detection of positioning errors, SVM, KNN, and XGBoost models showed good performance. As shown in Fig. 4, the AUC values obtained for the three classification models are 0.93, 0.93, and 0.94, respectively, indicating that radiomics analysis of EPID measurements performed excellently in the detection of clinically relevant positioning error.

The AUC values of direction classification were all larger than 0.70 , as shown in Fig. 5. This suggests radiomics analysis of EPID measurements is capable of detecting patient positioning errors in a specific direction. However, there were differences among the detectability of clinically relevant positioning errors in LR, SI, and AP directions. In the SI direction, the AUC values of SVM, KNN, and XGBoost were higher than 0.90. In the AP and LR directions, the highest AUC values were 0.80 and 0.76 , respectively.

\section{Discussion}

Our analysis revealed the potential for the use of radiomics and machine learning approaches in patient positioning error detection from EPID measurements with multifactorial error sources (positioning errors and linac mechanical errors). Anatomical changes, positioning errors,

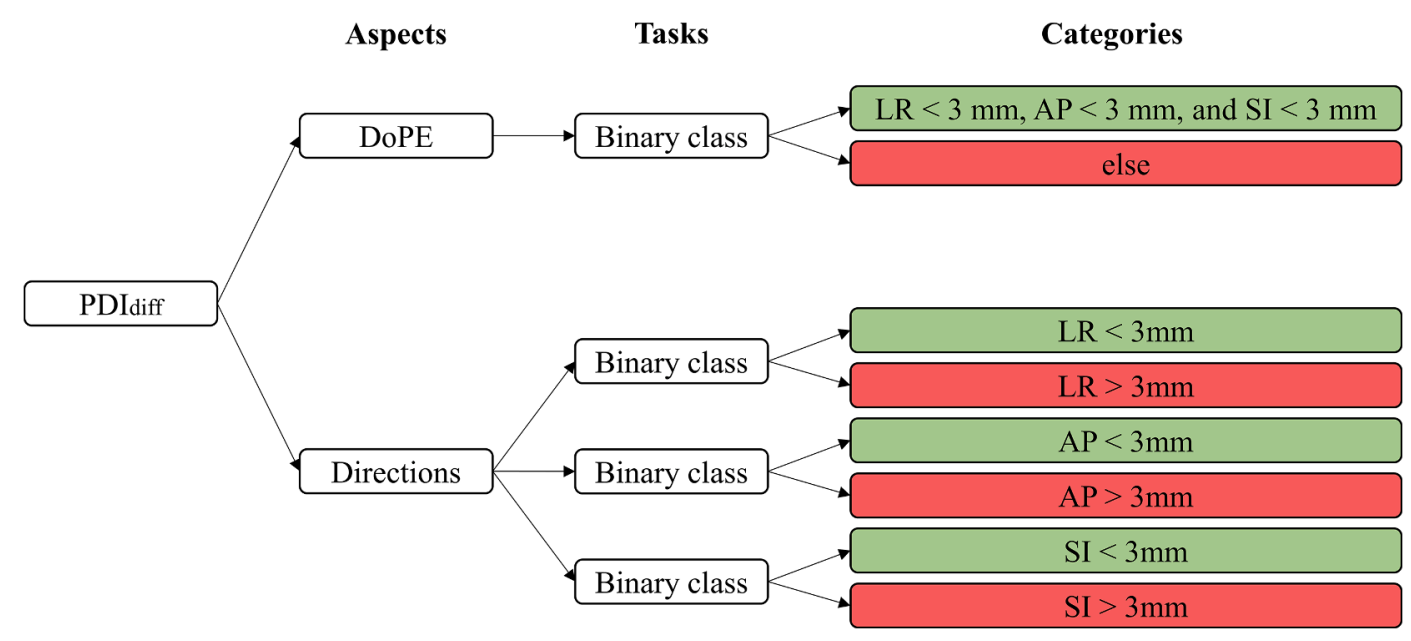

Fig. 2. Overview of the classification tasks and classification labels. DoPE refers to the detection of positioning errors. 


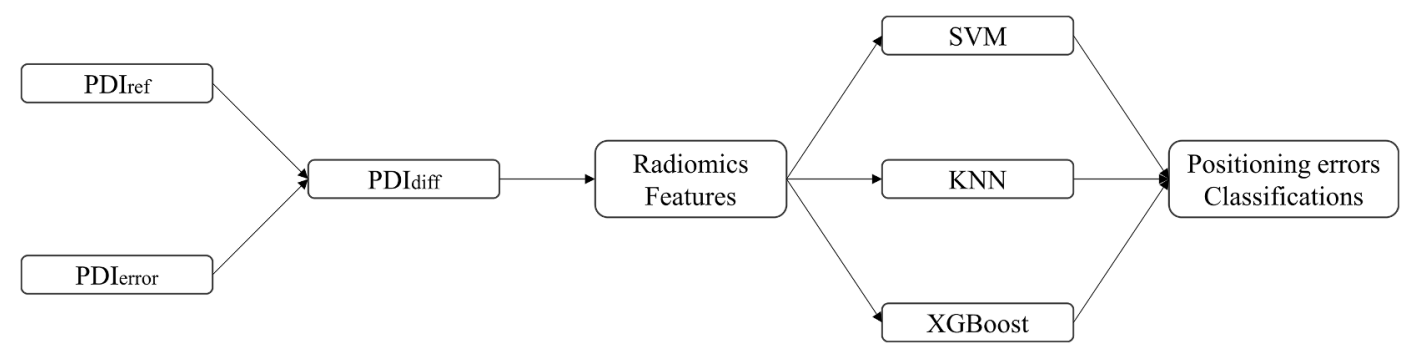

Fig. 3. The radiomics analysis workflow for positioning errors detection with EPID measurements.

Table 1

The F1-score of training, validation, and test data sets.

\begin{tabular}{lllll}
\hline Classification task (F1-score) & & Training & Validation & Test \\
\hline DoPE & SVM & 0.887 & 0.875 & 0.879 \\
& KNN & 0.999 & 0.887 & 0.866 \\
Direction (LR) & XGBoost & 0.939 & 0.878 & 0.871 \\
& SVM & 0.819 & 0.774 & 0.758 \\
& KNN & 1.000 & 0.716 & 0.692 \\
Direction (SI) & XGBoost & 0.886 & 0.744 & 0.713 \\
& SVM & 0.861 & 0.816 & 0.793 \\
Direction (AP) & KNN & 1.000 & 0.824 & 0.827 \\
& XGBoost & 0.995 & 0.835 & 0.820 \\
& SVM & 0.766 & 0.747 & 0.741 \\
& KNN & 0.999 & 0.741 & 0.760 \\
& XGBoost & 0.843 & 0.752 & 0.738 \\
\hline
\end{tabular}

DoPE refers to the detection of positioning errors.

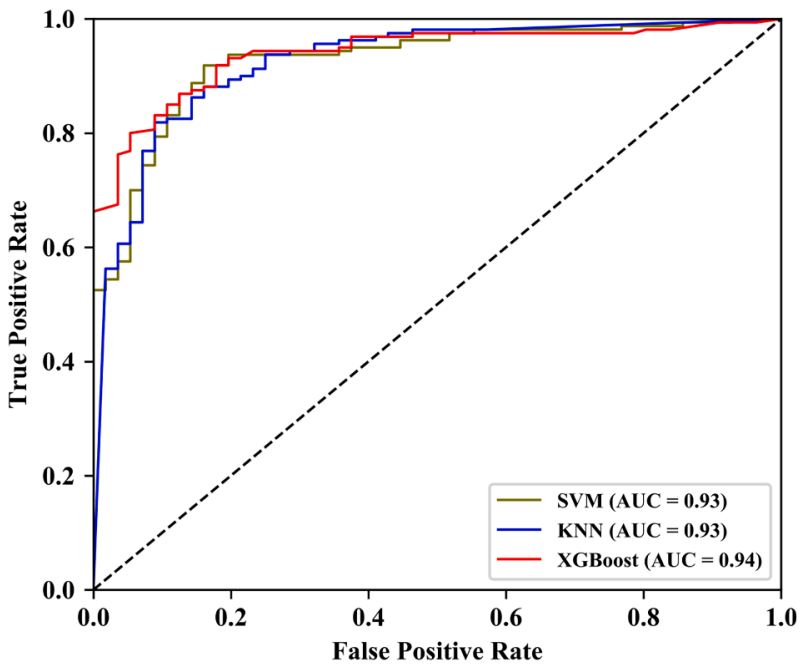

Fig. 4. The ROC curves of the detection of positioning errors.

and linac mechanical errors are the three main treatment error sources during radiotherapy delivery. For patients with thyroid-associated ophthalmopathy, anatomical changes are negligible. To approximate a clinical scenario, we simulated patient positioning errors and introduced actual linac mechanical errors as multifactorial error sources for EPID measurements. As discussed by Ma et al. [10], treatment errors are often introduced by positioning errors and anatomical changes rather than by linac mechanical errors. Therefore, in this study, we attempted to analyze the detectability of positioning errors from EPID measurements with multifactorial error sources.

Our results improved on those obtained by previous studies in that we were able to successfully apply the radiomic analysis of EPID measurements in detecting both the magnitude and direction of positioning errors. As reported by Zhuang et al. [3] and Hsieh et al. [4], conventional gamma analysis is limited to detecting the magnitude of

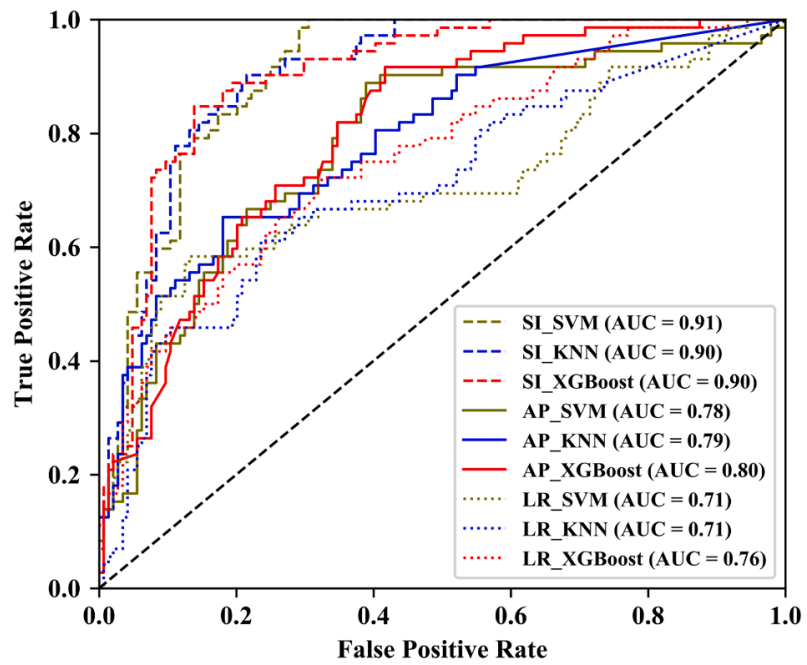

Fig. 5. The ROC curves of directions classifications of positioning errors.

positioning errors. Recently, Wolfs et al. [11] also explored the use of a learning-based approach in positioning error detection in lung cancer radiotherapy. Their model achieved 0.82 accuracy for classifying positioning errors exceeding $10 \mathrm{~mm}$ in a simulation study, whereas in our study we obtained F1-scores of above 0.86 in classifying positioning errors exceeding $3 \mathrm{~mm}$ (the detection of positioning errors). We also further investigated the detectability of the positioning error direction (direction classification).

Our SI direction classification results were better than our LR and AP direction classification results. A possible reason for this is that the positioning errors in the SI direction are completely reflected at each gantry rotation angle because this direction is perpendicular to the gantry rotation panel. Conversely, the positioning errors in the LR and AP directions only affect the transmission dosimetry of the EPID at partial gantry rotation angles. We also found that, although the direction classifications were less accurate than the positioning classifications, the performances of the SVM, KNN, and XGBoost in obtaining these were comparable. This indicates that the primary limitation of our direction classification approach is the features that it can extract.

Although our cohort of 40 patients must be considered a small-sized sample, the differences between the validation and test data set results were negligible. This indicates the number of participants we enrolled was sufficient in terms of fitting the detection of positioning errors and direction classification. One possible reason for this is that the lesions sizes and locations of thyroid-associated ophthalmopathy patients are relatively fixed, which was in fact our reason for including only patients with thyroid-associated ophthalmopathy in the study. Transferring this method to other treatment sites will be the subject of our future work.

The primary limitation of this study was that the impacts of machine output and beam uniformity change over time were not evaluated. Machine output has been proven to be a high degree of linearity with the EPID measurements by Zhuang et al. [4]. This implies that the machine 
output error can be eliminated via EPID calibration. On the other hand, variation of beam uniformity is complex [20] and long-term studies will be required to evaluate its impact on EPID measurements. Besides, the simulated translation positioning errors failed to cover all the possibilities of positioning errors. However, this study simulated combinations of translation positioning errors in LR, SI, and AP axis, and obtained satisfactory results in direction classification.

\section{Conclusions}

In this study, we demonstrated that combined radiomics and machine learning approaches are capable of detecting the magnitude and directions of positioning errors from EPID measurements with multifactorial error sources. High performance is achievable using a smallsize sample for patients with thyroid-associated ophthalmopathy. This study is a further step toward the practical implementation of machine learning-based positioning error detection using EPID measurements.

\section{Declaration of Competing Interest}

The authors declare that they have no known competing financial interests or personal relationships that could have appeared to influence the work reported in this paper.

\section{Acknowledgements}

This work was supported by the National Natural Science Foundation of China (Grant No. 81472807, 81972848, and 11905150), and the Sichuan Science and Technology Program (No. 2021YFS0143 and 2018HH0099).

\section{Appendix A. Supplementary data}

Supplementary data to this article can be found online at https://doi. org/10.1016/j.ejmp.2021.08.014.

\section{References}

[1] Ezzell GA, Galvin JM, Low D, Palta JR, Rosen I, Sharpe MB, et al. Guidance document on delivery, treatment planning, and clinical implementation of IMRT: report of the IMRT Subcommittee of the AAPM Radiation Therapy Committee. Med Phys 2003;30(8):2089-115. https://doi.org/10.1118/1.1591194.

[2] van Elmpt W, McDermott L, Nijsten S, Wendling M, Lambin P, Mijnheer B. A literature review of electronic portal imaging for radiotherapy dosimetry. Radiother Oncol 2008;88(3):289-309. https://doi.org/10.1016/j. radonc.2008.07.008.

[3] Li Y, Zhu J, Shi J, Chen L, Liu X, Leal A. Investigating the effectiveness of monitoring relevant variations during IMRT and VMAT treatments by EPID-based
3D in vivo verification performed using planning CTs. PLoS ONE 2019;14(6): e0218803. https://doi.org/10.1371/journal.pone.0218803.

[4] Zhuang AH, Olch AJ. Sensitivity study of an automated system for daily patient QA using EPID exit dose images. J Appl Clin Med Phys 2018;19(3):114-24. https:// doi.org/10.1002/acm2.2018.19.issue-310.1002/acm2.12303.

[5] Hsieh ES, Hansen KS, Kent MS, Saini S, Dieterich S. Can a commercially available EPID dosimetry system detect small daily patient setup errors for cranial IMRT/ SRS? Pract Radiat Oncol 2017;7(4):e283-90. https://doi.org/10.1016/j. prro.2016.12.005.

[6] Miften M, Olch A, Mihailidis D, Moran J, Pawlicki T, Molineu A, et al. Tolerance limits and methodologies for IMRT measurement-based verification QA: Recommendations of AAPM Task Group No. 218. Med Phys 2018;45(4):e53-e83. https://doi.org/10.1002/mp.12810.

[7] Potter NJ, Mund K, Andreozzi JM, Li JG, Liu C, Yan G. Error detection and classification in patient-specific IMRT QA with dual neural networks. Med Phys 2020;47(10):4711-20. https://doi.org/10.1002/mp.v47.1010.1002/mp.14416.

[8] Nyflot MJ, Thammasorn P, Wootton LS, Ford EC, Chaovalitwongse WA. Deep learning for patient-specific quality assurance: Identifying errors in radiotherapy delivery by radiomic analysis of gamma images with convolutional neural networks. Med Phys 2019;46(2):456-64. https://doi.org/10.1002/mp.2019.46. issue-210.1002/mp.13338.

[9] Wootton LS, Nyflot MJ, Chaovalitwongse WA, Ford E. Error detection in intensitymodulated radiation therapy quality assurance using radiomic analysis of gamma distributions. Int J Radiat Oncol Biol Phys 2018;102(1):219-28. https://doi.org/ 10.1016/j.ijrobp.2018.05.033.

[10] Ma C, Wang R, Zhou S, Wang M, Yue H, Zhang Y, et al. The structural similarity index for IMRT quality assurance: Radiomics-based error classification. Med Phys 2021;48(1):80-93. https://doi.org/10.1002/mp.v48.110.1002/mp.14559.

[11] Wolfs CJA, Canters RAM, Verhaegen F. Identification of treatment error types for lung cancer patients using convolutional neural networks and EPID dosimetry. Radiother Oncol 2020;153:243-9. https://doi.org/10.1016/j.radonc.

[12] Gillies RJ, Kinahan PE, Hricak H. Radiomics: images are more than pictures. They Are Data. Radiology 2016;278(2):563-77. https://doi.org/10.1148/ radiol.2015151169.

[13] van Griethuysen JJM, Fedorov A, Parmar C, Hosny A, Aucoin N, Narayan V, et al. Computational radiomics system to decode the radiographic phenotype. Cancer Res 2017;77(21):e104-7. https://doi.org/10.1158/0008-5472.CAN-17-0339.

[14] Li YJ, Luo Y, He WM, Li P, Wang F. Clinical outcomes of graves' ophthalmopathy treated with intensity modulated radiation therapy. Radiat Oncol 2017;12(1):171. https://doi.org/10.1186/s13014-017-0908-7.

[15] de Deus Cardoso CC, Giordani AJ, Wolosker ÂMB, Souhami L, Manso PG, Dias RS, et al. Protracted hypofractionated radiotherapy for Graves' ophthalmopathy: A pilot study of clinical and radiologic response. Int J Radiat Oncol Biol Phys 2012;82 (3):1285-91. https://doi.org/10.1016/j.ijrobp.2010.09.015.

[16] White DR, Buckland-Wright JC, Griffith RV, Rothenberg LN, Showwalter CK, Williams G, et al. 4. Phantoms in radiotherapy. Rep Internat Comm Radiat Units Measur 1992;os-25(1):21-5. https://doi.org/10.1093/jicru os25.1.21.

[17] Baeza JA, Wolfs CJA, Nijsten SMJJG, Verhaegen F. Validation and uncertainty analysis of a pre-treatment 2D dose prediction model. Phys Med Biol 2018;63(3): 35033. https://doi.org/10.1088/1361-6560/aa9d0c.

[18] Virtanen P, Gommers R, Oliphant TE, Haberland M, Reddy T, Cournapeau D, et al. Author correction: SciPy 1.0: fundamental algorithms for scientific computing in Python. Nat Methods 2020;17(3):352. https://doi.org/10.1038/s41592-020-07725 .

[19] Chen T, Guestrin C. XGBoost: A scalable tree boosting system. the 22nd ACM SIGKDD international conference. 2016.

[20] Calvo JF, Eraso A, Garrido L, Casals J. 459 Daily constancy checks of flatness, symmetry and output for photon beams using a commercial electronic portal image device (EPID). Radiother Oncol 2005;76:S198-9. https://doi.org/10.1016/S01678140(05)81435-5. 